\title{
FROM SD TO HD TELEVISION: EFFECTS OF H.264 DISTORTIONS VERSUS DISPLAY SIZE ON QUALITY OF EXPERIENCE
}

\author{
Stéphane Péchard, Mathieu Carnec, Patrick Le Callet, Dominique Barba
}

\author{
Institut de recherche en communication et cybernétique de Nantes (IRCCyN) \\ Équipe Image et vidéocommunication - UMR CNRS 6597 \\ Polytech'Nantes, rue Christian Pauc, La Chantrerie \\ BP 50609, 44306 Nantes, Cedex 3, France \\ $\{$ stephane.pechard; mathieu.carnec ; patrick.lecallet ; dominique.barba $\} @$ univ-nantes.fr
}

\begin{abstract}
High Definition Television (HDTV) is the new broadcasting system designed to take the place of Standard Definition Television (SDTV) at home in the near future. This system requires modification of many features in the broadcasting chain with an overall objective of reaching a noticeably higher quality of experience. Since broadcasters desire a high level of service acceptability, they require efficient measurements of quality of experience. The purpose of this paper is to provide such measurements concerning the noticeable artifacts in H.264 distortions over a range of display sizes and comparing HDTV to SDTV. A subjective characterization of some HDTV quality of experience aspects is proposed and the results are discussed.
\end{abstract}

Index Terms - HDTV, Communication equipment testing, Technology assessment.

\section{INTRODUCTION}

HDTV services have already been introduced in USA, Australia, Japan, Canada and Korea. They are expected in Europe and China in 2006. Such systems are motivated by psychophysical considerations. Subjective tests [1,2] have shown that the ideal distance to watch moving pictures is about three times the screen's height. Therefore, in order to avoid perception of pictures defects when standing at this distance, HDTV relies on a wider display screen and a higher source definition.

First generation HDTV is broadcast using MPEG-2 in two definitions: $1920 \times 1080$ in interlaced mode (named 1080i) and $1280 \times 720$ in progressive mode $(720$ p). Subjective tests tend to suggest that $720 \mathrm{p}$ is visually of better quality than 1080i [3] for video with rather high motion. But as most video cameras are currently in $1080 \mathrm{i}$ format, this is more likely to be used in production. Effectively, a larger image would be more appealing commercially and consumers would probably be influenced by the misnomer "the larger the image the better" and prefer $1080 \mathrm{i}$ over $720 \mathrm{p}$. Second generation HDTV is developed with the same definitions but using an H.264/MPEG-4 AVC compression standard. Quality metrics should therefore be defined for $\mathrm{H} .264$ specificities. Third generation HDTV will use $1920 \times 1080$ definition in progressive mode. At present however, too few capture materials are available in this format. Moreover, important investments have been made in the two first generations, so they must make a profit before new investments are made.

Since a user's quality expectations are increased in HDTV and display screens are larger, broadcasters have to allocate higher bitrates for HDTV than for SDTV. Therefore this study can assist in determining the service satisfaction threshold. But there are very few references on HDTV quality measurements. This paper attempts to fill this gap in part.

Previous work investigated the design of HDTV subjective quality assessment protocols [4]. Since HDTV provides a new experience for the end-user, his/her habits in terms of television viewing are expected to change. The observation distance has been particularly studied. Tests have been conducted to evaluate viewing differences between HDTV and SDTV. Observers are likely to prefer the larger image which is proposed by HDTV but if too many distortion artifacts are present, some of them may prefer SDTV. These tests have been designed to determine the preference threshold between HDTV and SDTV and to evaluate the effect of a larger image.

The next section describes the subjective tests performed. Particular attention is given to material, observers and protocol. In Section 3, experimental results are given along with a discussion. The paper is concluded in Section 4

\section{SUBJECTIVE TESTS}

\subsection{Video material}

Four ten-second long 1080i HDTV sequences from SVT research [3] have been used. Example frames are presented in Figure 1 Sequences have been distorted through the use of H.264 JM reference software [5]. Seven bitrates have been produced per HD sequence. Bitrates differ from one sequence 


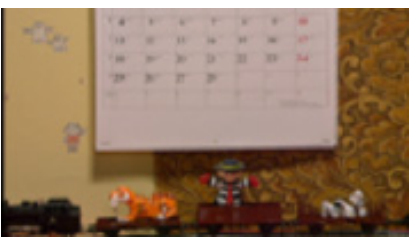

(a) New Mobile \& Calendar: indoor, slow traveling.

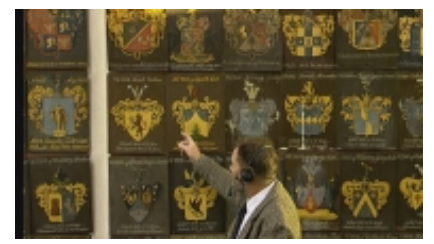

(c) Knightshields: indoor, traveling and zoom.

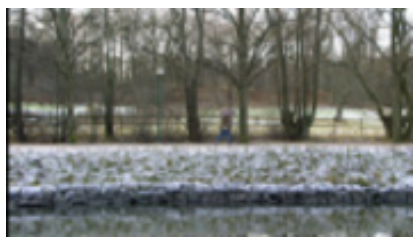

(b) Parkrun: outdoor, multiple planes traveling.

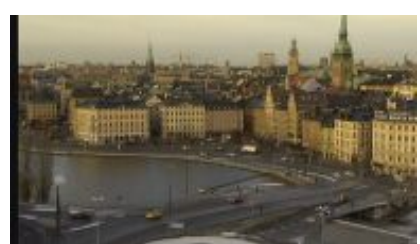

(d) Stockholm Pan: outdoor, panning.
Fig. 1. Example of HDTV sequences usable for test protocol.

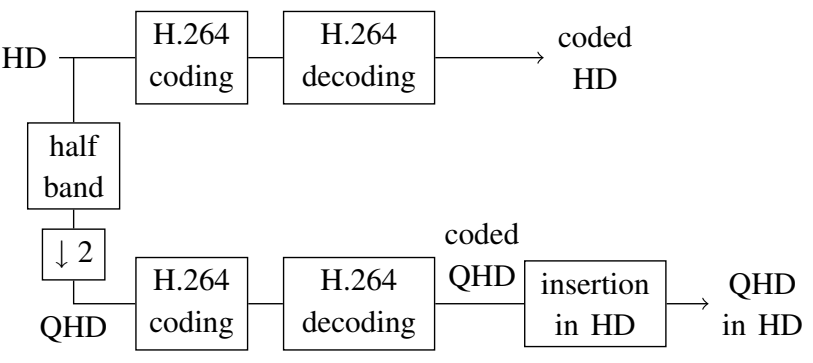

Fig. 2. Subjective tests protocol sequences obtaining scheme.

to another and are presented in Table 1 . The selection of these bitrates has been done during preliminary tests by the authors in order to cover the useful quality range from a poor to an excellent visual quality.

SDTV sequences are computed from these HD sequences through a half band filtering followed by sub-sampling by a factor of 2 (both along horizontal and vertical directions). Both fields are decimated separately before reconstitution in progressive format. A general scheme is presented in Figure 2. This results in an approximation of SD 576i sequences (the actual definition is $960 \times 540$ ). This technique is motivated by the fact that this definition is very close to "real 16/9" SDTV $(1024 \times 576)$ and no interpolation is required. Furthermore, this results in a half-height video (QHD in figure 2, for Quarter HD), which can satisfy both recommended distances for SD $(D=6 H)$ and HD $(D=3 H), H$ being the video's height [1, 2, 6, 7].

As with HD sequences, SD videos have been encoded using the H.264 reference software [5], with the same parameters. MPEG-2 has not been used in order to avoid introducing another difference in the comparison. Each SD sequence has been encoded with two bitrates corresponding to two common

\begin{tabular}{|c|c|}
\hline Sequence & HDTV Bitrates (Mbps) \\
\hline \hline New Mobile \& Calendar & $2.25 ; 2.5 ; 3.15 ; 4 ; 5 ; 7 ; 10$ \\
\hline Parkrun & $8 ; 12 ; 16 ; 18 ; 20 ; 24 ; 28$ \\
\hline Knightshields & $2.25 ; 3 ; 4 ; 5 ; 6 ; 7 ; 8$ \\
\hline Stockholm Pan & $1.625 ; 1.875 ; 2.25 ; 3 ; 3.6 ; 4 ; 6$ \\
\hline \hline Sequence & SDTV Bitrates $\left(Q_{60}, Q_{80}\right)$ \\
\hline \hline New Mobile \& Calendar & $1.8 ; 3$ \\
\hline Parkrun & $5.3 ; 9$ \\
\hline Knightshields & $1.6 ; 3$ \\
\hline Stockholm Pan & $1.2 ; 1.8$ \\
\hline
\end{tabular}

Table 1. Chosen bitrates (in Mbps) per video sequence.

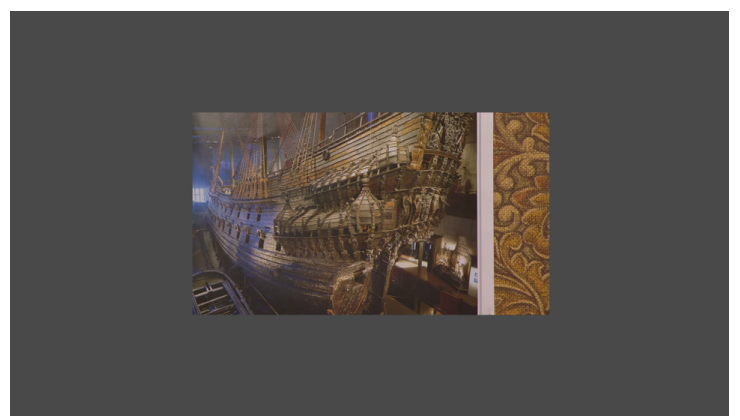

Fig. 3. Example of a SD image inserted in a HD image.

SD broadcast qualities. They have been chosen to be representative of an excellent $\left(Q_{80}\right)$ and of a rather good $\left(Q_{60}\right)$ subjective visual quality, respectively. It means to get scores of around 80 and 60 on a continuous subjective quality scale of 1 to 100 . Selected bitrates are given in Table 1 These will be compared to the seven different HD quality levels. To avoid screen flickering and screen's manual switching between HD and SD, SD videos have been inserted at the center of an HD video with gray background. An example of a SD image inserted in a HD image is presented in Figure 3.

\subsection{Test room material}

Tests have been performed in a specific showroom. Lightning conditions, display parameters and observation distances have been precisely measured as required in recommendations [6, 7]. The HDTV screen used was a CRT JVC DTV 1910CG which can display 1080i sequences. Its height $D$ equals $20.5 \mathrm{~cm}$, which means that an observer is positioned at a distance of about $61.5 \mathrm{~cm}$. Uncompressed 1080i video sequences were played with a V1-UHD player from the Dorémi company.

\subsection{Protocol}

Specific protocols have been designed for these tests since no such experiments have been normalized yet. The pro- 


\begin{tabular}{|c|c|}
\hline Caption to choose & Value stored \\
\hline \hline I prefer much more A than B & +3 \\
\hline I prefer more A than B & +2 \\
\hline I prefer a little more A than B & +1 \\
\hline I have no preference & 0 \\
\hline I prefer a little less A than B & -1 \\
\hline I prefer less A than B & -2 \\
\hline I prefer much less A than B & -3 \\
\hline
\end{tabular}

Table 2. Comparison scale for comparison test protocol.

tocol utilised is derived from the comparative method with adjectival categorical judgment described in BT.500-11 ITU Recommendation [6]. Before the actual comparison test, observers were simply asked to determine their preferred observation distance from the HDTV screen. This information is important since some distortions may be visible at a certain distance and invisible at another one. Furthermore, no precision were given about the recommended observation distance. Thus, people mostly determined their preferred distance with their own television culture. The criterion for determination is observer's comfort of visualization. Then he/she was asked to take place at the recommended distance of $3 H$.

Series of comparison tests were made of the presentations. A presentation consisted of one or several visualizations of two video sequences labeled "video A" and "video B". HD and SD videos were assigned letter A or B randomly. A visualization was the viewing of both videos A and B. During each visualization, the observer compared $\mathrm{A}$ and $\mathrm{B}$. After an initial visualization, he/she could replay any video as much as he/she wanted before voting. This random access increases reliability, as shown in the EBU's SAMVIQ methodology [8]. After each presentation, the observer had to report the existence and direction of perceptible differences he/she perceived. The comparison scale used is shown in Table 2 Values stored were not shown to the observer. Words associated with quality such as better or worse were not used. This way, the observer reports his/her global preference, not through a quality criterion.

\subsection{Observers}

Every observer was first checked for color blindness with Ishihara test [9] and for acuity with Monoyer's plates [10]. People with at least one error in Ishihara's test or less than $9 / 10$ in Monoyer's test were rejected. 21 people took part in these tests (a minimum of 15 is recommended [6]).

Observers were mostly male students in their mid twenties. All are familiar with SDTV and cinema but not with HDTV. When asked about the difference between standing at their preferred distance and at the recommended $3 \mathrm{H}$, most of them were not specifically disturbed, but some indicated they felt too close to the screen and needed to stand back.

\section{RESULTS}

\subsection{User's experience}

When users were asked to determine their ideal HDTV observation distance, all went farther than $3 H$. Average viewing distance measured was $161 \mathrm{~cm}$ (which is about $8 H$ ) with a standard deviation of $36 \mathrm{~cm}$. This result is due to the habit of SDTV usual observation distance. People have a certain television viewing culture, and without informing them that HDTV is supposed to be watched closer, they don't adapt their habits to the new media.

It is also interesting to notice that when positioned at $3 \mathrm{H}$, most observers expressed the feeling that there was too much information to process, therefore observers often needed to stand back in order to consider so much information. The screen was so close that motion in the peripheral area of the retina was disturbing for many.

The replay option was not used much, as every sequence was played 1.15 times on average.

\subsection{Image size and distortions effects}

PSNRs of the sequences have been computed to measure the artifact importance. PSNR of a sequence is taken as the mean PSNR along all frames. For each sequence and each SDTV bitrate, $\triangle P S N R$ is defined as $\triangle P S N R=P S N R_{H D}-$ $P S N R_{S D}$. It measures the artifact difference between HD and SD. Figure 4 presents the mean opinion score (MOS) as a function of $\triangle P S N R$ with $95 \%$ confidence intervals for the Stockholm Pan sequence. This figure indicates that users prefer HD over SD depending on the artifact importance difference. Results with other sequences are similar. The arrow indicates the $\triangle P S N R_{0}$ value, which equals the $\triangle P S N R$ value for $M O S=0$. If it is negative, it means that when users have no preference between HD and SD, the HD sequence contains more artifacts. In Table 3 , the $\triangle P S N R_{0}$ and $P S N R_{H D}$ values are given for each sequence. These values have been obtained by linear interpolation.

Some conclusions can be drawn from Figure 4 and Table 3. First, with a decreasing $\triangle P S N R, \mathrm{SD}$ is preferred to $\mathrm{HD}$, which is the expected behavior. Then, when HD is compared to SD of a good visual quality $\left(Q_{80}\right), \triangle P S N R$ is always negative. This means that HD may contain more artifacts and still obtain the same preference level. Therefore, the impact of the large screen effect is predominant and rules the observer's preference in this case. On the contrary, when HD is compared to $\mathrm{SD}$ of lower but still rather good quality $\left(Q_{60}\right)$, this effect decreases and is even reversed in some cases with $\triangle P S N R_{0}>0$. Distortions in HD become more prevalent and disturbing. Here observers tend to prefer SD implying distortions on a large image is more disturbing than on a small one. In this case, the effect of distortions is predominant over the large image effect. 


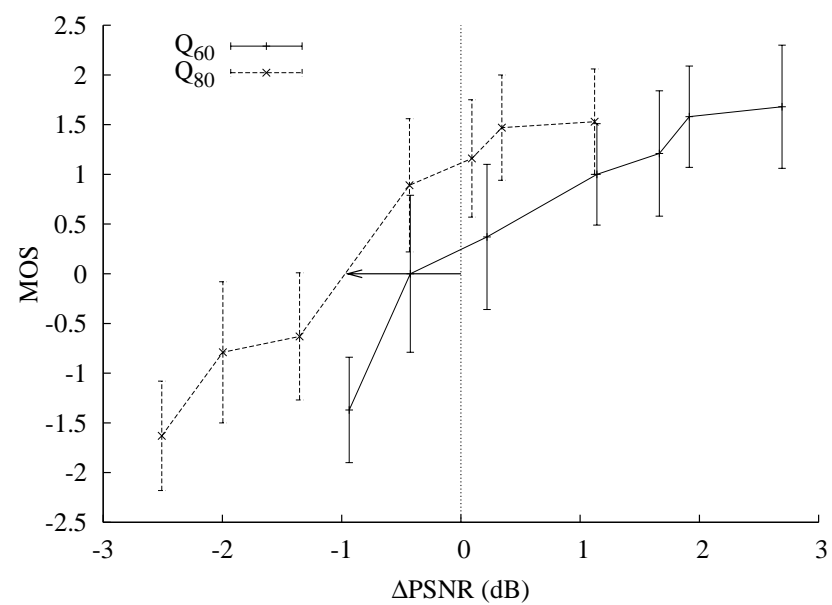

Fig. 4. $\triangle P S N R$ values in function of MOS for Stockholm Pan sequence.

\begin{tabular}{|c|c|c|}
\hline Sequence $Q_{80}$ & $\triangle P S N R_{0}$ & $P S N R_{H D}$ \\
\hline New Mobile \& Calendar & -0.65 & 37.60 \\
\hline Parkrun & -2.65 & 34.36 \\
\hline Knightshields & -0.46 & 37.81 \\
\hline Stockholm Pan & -0.97 & 37.81 \\
\hline \hline Sequence $Q_{60}$ & $\Delta P S N R_{0}$ & $P S N R_{H D}$ \\
\hline New Mobile \& Calendar & 1.27 & 37.39 \\
\hline Parkrun & -0.87 & 33.23 \\
\hline Knightshields & 1.5 & 37.00 \\
\hline Stockholm Pan & -0.43 & 36.78 \\
\hline
\end{tabular}

Table 3. $P S N R_{H D}$ and $\triangle P S N R_{0}$ for both SD sequences.

\section{CONCLUSION}

In this paper, subjective tests and results have been presented. These tests were performed in order to characterize differences in quality of experience between High Definition Television (HDTV) and Standard Definition Television (SDTV). Results show that HDTV is a change in customer's habits. People have cultural prejudices about television and may need information to change it.

Two important effects have been identified as critical in switching from SDTV to HDTV. Comparison tests prove that distortions and a larger image both influence the observer's preference. Results tend to show that distortions are the predominant factor when HDTV sequences are compared with low quality SDTV. However, in the case of high quality SDTV, the image effect becomes more important. This means that image size is a factor of visual comfort when images are only slightly distorted. But larger image size becomes a drawback when the level of distortions increases. Observers then prefer standard definition, as this reduces the visual impact of the distortions. Therefore HDTV has to reach a high level of quality to be successfully adopted by consumers.

\section{ACKNOWLEDGMENT}

This work is supported by HD4U European project. Its aim is to study the best conditions to deploy HDTV in Europe. Several actors from consumer devices industries (Philips, Thomson, etc.) and broadcasters (TF1, Euro1080) are involved. Authors would also like to thank SVT for the freely available HDTV sequences, Thomson for the HDTV screen and Arnaud Tirel and Sylvain Tourancheau for their assistance in performing the subjective tests described in this paper.

\section{REFERENCES}

[1] Tetsuo Mitsuhashi, "Fundamental requirements for High-definition television systems - Scanning specifications and picture quality," NHK technical monograph, pp. 21-26, NHK, 1982.

[2] Ichiro Yuyama, "Fundamental requirements for Highdefinition television systems - Large-screen effects," NHK technical monograph, pp. 14-20, NHK, 1982.

[3] SVT, "Overall-quality assessment when targeting Wide XGA flat panel displays," Tech. Rep., SVT corporate development technology, 2002, ftp://ftp.ldv.etechnik.tu-muenchen.de/pub/test_sequences/.

[4] Stéphane Péchard, Sylvain Tourancheau, Patrick Le Callet, Mathieu Carnec, and Dominique Barba, "Towards Video Quality Metrics for HDTV," in Proceedings of the Second International Workshop on Video Processing and Quality Metrics for Consumer Electronics, VPQM2006, Scottsdale, 2006.

[5] Joint Video Team (JVT), "H.264/Advanced Video Coding reference software version 10.1," 2005, http://iphome.hhi.de/suehring/tml/.

[6] ITU-R BT. 500-11, "Methodology for the subjective assessment of the quality of television pictures," Tech. Rep., International Telecommunication Union, 2004.

[7] ITU-R BT. 710-4, "Subjective assessment methods for image quality in high-definition television," Tech. Rep., International Telecommunication Union, 1998.

[8] Jean-Louis Blin, "SAMVIQ - Subjective assessment methodology for video quality," Tech. Rep. BPN 056, EBU Project Group B/VIM Video in Multimedia, 2003.

[9] Shinobu Ishihara, "Tests for colour-blindness," Kanehara Shuppen Company, Ltd., Tokyo, Japan, 1967.

[10] Ferdinand Monoyer, "Échelle typographique pour la détermination de l'acuité visuelle," Académie des Sciences, Comptes rendus, vol. 80, no. 113, 1875. 